\title{
Long-term optical activity of the microquasar V4641 Sgr
}

\author{
Vojtěch Šimon \\ Astronomical Institute, Academy of Sciences of the Czech Republic, 25165 Ondrejov, Czech \\ Republic \\ E-mail: simoneasu.cas.cz
}

\section{Arne Henden}

AAVSO, 49 Bay State Road, Cambridge, MA 02138, USA

E-mail: arne@aavso.org

We present an analysis of the optical activity of the microquasar V4641 Sgr using the visual and photographic data. We analyze four smaller (echo) outbursts that followed the main outburst (1999). Their mean recurrence time $T_{\mathrm{C}}$ is 377 days, with a trend of a decrease. We interpret the characteristic features of the recent activity of V4641 Sgr (the narrow outbursts separated by a long quiescence, a clear trend in the evolution of their $T_{\mathrm{C}}$ ) as the thermal instability of the accretion disk operating in dwarf novae and soft X-ray transients. We argue that the luminosity of four echo outbursts is too high to be explained by the thermal emission of the accretion disk. We interpret them as due to the thermal instability, in which the outburst gives rise to a jet radiating also in the optical. This supports the finding by Uemura et al., PASJ 54, L79 (2002). The pre-outburst observations (1964-1967) reveal ongoing activity of the system. It displays low-amplitude fluctuations on the timescale of several weeks, independent on the orbital phase. In addition, a larger brightening which lasted for several tens of days and occurred from the level of brightness higher than in other years can be resolved.

VII Microquasar Workshop: Microquasars and Beyond

September 1 - 5, 2008

Foca, Izmir, Turkey 


\section{Introduction}

V4641 Sgr is a binary system consisting of the black hole accretor and a B9III donor at the distance of 7-12 kpc [8]. Its orbital period is $P_{\text {orb }}=2.81728 \mathrm{~d}$ [2]. It is the optical counterpart of the X-ray transient SAX J1819.3-2525 [13]. The first outburst was detected on a photographic plate in 1978 [1]. The major outburst which occurred in September 1999 was observed in Xrays, optical, and radio. Radio structures suggest superluminal jet ejection [5]. This event was preceded by an increased X-ray activity starting already in February 1999 (e.g. [7]). The major outburst was associated with an episode of super-Eddington accretion onto the black hole. During outburst, extended optically thick envelope/outflow has been formed [9]. Several smaller outbursts followed the 1999 event in the subsequent years. The continuum brightened during these events [6]. P Cygni profiles indicated rapid changes in matter ejection. Rapid optical fluctuations indicate that the emission from an inner accretion region significantly contributes to the optical flux. It was interpreted as cyclo-synchrotron emission associated with magnetic flares [14].

\section{Data analysis}

The recent photometric history of V4641 Sgr in the optical passband was investigated using the visual data from the American Association of Variable Star Observers (AAVSO) International database [4]. Since our analysis was focused on the long-term activity with large-amplitude events, the accuracy of these data proved to be quite sufficient. This lightcurve contained the 1999 main outburst and four smaller echo outbursts (Figure 1a). Each of them is defined by multiple observations. The echo outbursts display multi-peak structure and accumulation of the points near the peak magnitude (Figures 1c and 1d).

The evolution of the recurrence time $T_{\mathrm{C}}$ of the outbursts was investigated using the method of the $\mathrm{O}-\mathrm{C}$ residuals in a similar way as in $[15,11]$ (Figure 1b). It can be seen that the $\mathrm{O}-\mathrm{C}$ values of the echo outbursts display a clearly defined trend in their time evolution, with the superposed outburst-to-outburst fluctuations having a significantly smaller amplitude. In the case of one missing echo outburst in the epoch $E=-2$ (falling into the seasonal gap) also the main outburst follows the extrapolation of the $\mathrm{O}-\mathrm{C}$ curve of the echo outbursts.

In order to compare the peak magnitude of the echo outburst with that of soft X-ray transients (SXTs), the relation of the absolute visual magnitude $M_{\mathrm{V}}$ of SXTs at the outburst maximum vs. $P_{\text {orb }}$ was investigated (Figure 1e). The parameters of 10 SXTs come from [10]. Three values of the distance to V4641 Sgr were considered.

Photometric history of V4641 Sgr in the epoch long time before the 1999 main outburst was studied on the photographic plates from the archive of the Bamberg Observatory. These plates were blue-band sensitive, which is similar to the $B$ filter. The exposure time was $60 \mathrm{~min}$. These observations covered the years 1964-1967 (Figure 1f). The points are connected by line in densely covered segments. Typical uncertainty is $0.05 \mathrm{mag}$. Magnitudes are displayed with respect to the star GSC 0684802768. The statistical distribution of the brightness is displayed in Figure 1g. Notice the prevailing skewness toward the higher brightness. The data were folded with the orbital phase according to the ephemeris of [2]: Min I $=2451764.298+2.81728 \mathrm{E}$. It was found that the orbital modulation is precluded by the long-term brightness variations (Figure 1h). 

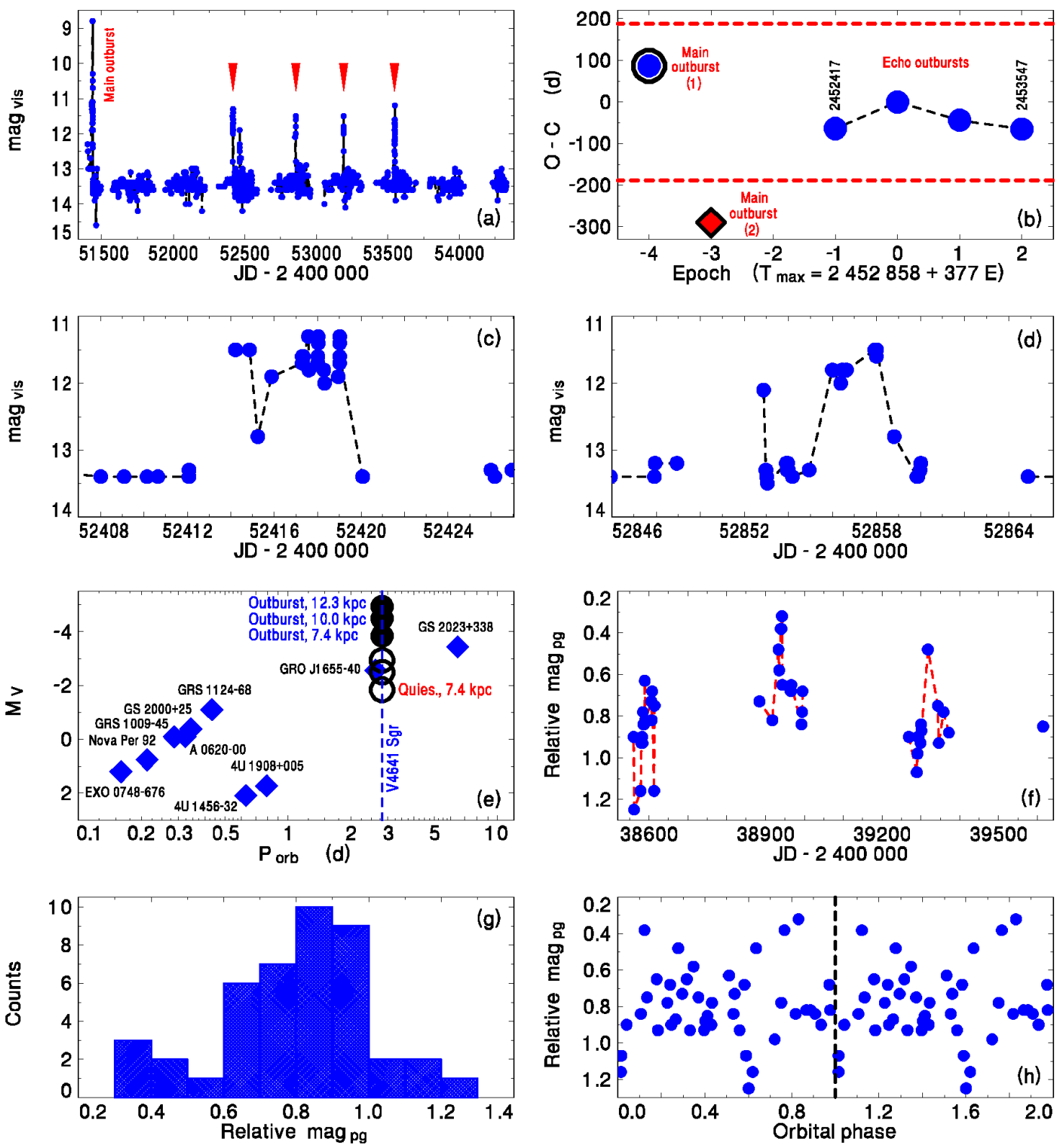

Figure 1: (a) The AVVSO visual light curve of V4641 Sgr. The peak of the 1999 main outburst is near the left hand edge of the plot. The echo outbursts are marked by arrows. (b) $\mathrm{O}-\mathrm{C}$ curve for $T_{\mathrm{C}}$ of the main and echo outbursts. Two positions of the main outburst are shown: (1) two missing echo outbursts; (2) one missing echo outburst. Dashed horizontal lines represent the length of the reference period of $377 \mathrm{~d}$ to show the relatively small scatter of $T_{\mathrm{C}}$ of the echo outbursts. (c, d) Examples of the echo outbursts. (e) The absolute visual magnitude $M_{\mathrm{V}}$ of SXTs at the outburst maximum vs. $P_{\text {orb }}$ (filled diamonds). The parameters of 10 SXTs, except for V4641 Sgr, are from [10]. Closed circles denote V4641 Sgr at the maximum of the echo outburst. Open circles mark V4641 Sgr in quiescence. Three values of the distance are assumed. (f) The photographic light curve of V4641 Sgr from the Bamberg plates (1964-1967) (relative mag). (g) Statistical distribution of the brightness in the Bamberg data. (h) The Bamberg data folded with the orbital period. See text for details. 


\section{Results}

We present an analysis of the optical activity of V4641 Sgr on the timescale of years.

The mean $T_{\mathrm{C}}$ of four echo outbursts that followed the main outburst is 377 days, with a trend of a decrease. The evolution of their $T_{\mathrm{C}}$ is analogous to that in outbursts of dwarf novae (e.g. [11]) and some SXTs (e.g. [12]). If one echo outburst in $E=-2$ was missed due to a seasonal gap, the main outburst nicely fits the evolution of $T_{\mathrm{C}}$ of the echo outbursts.

The accumulation of the data points near the peak magnitude of the echo outburst implies that these events do not consist of a long series of short, narrow peaks starting from the quiescent level of brightness.

The optical luminosity of the echo outbursts is too high to be explained by the thermal emission of the accretion disk analogous to that in SXTs of a comparable $P_{\text {orb }}$. The very luminous donor of V4641 Sgr outshines the thermal emission of the disk even in outburst. The morphology of the echo outbursts shows that their optical lightcurves decay very steeply, faster than what corresponds to the thermal timescale of the accretion disk in the system with $P_{\text {orb }}=2.8 \mathrm{~d}$, provided that the mass exchange occurs via Roche lobe overflow. The flat-top profile is inconsistent with a single expanding bubble emitting synchrotron radiation, but can be explained by a jet or a series of successive bubbles. The very short duration of the echo outburst resembles the radio flares observed during the X-ray outburst of the microquasar GRO J1655-40 [3].

We interpret the characteristic features of the recent activity of V4641 Sgr (the narrow outbursts separated by a long quiescence, a clear trend in the evolution of $T_{\mathrm{C}}$ ) as the thermal instability of the accretion disk operating in dwarf novae and SXTs. The intense optical emission of the echo outbursts in V4641 Sgr is due to a jet radiating cyclo-synchrotron emission [14]. The thermal emission of the disk itself remains outshined by the donor even in outburst. This scenario also explains why no other echo outburst was observed in V4641 Sgr. The thermal instability gives rise to the cyclically repeating outbursts, but if no jet radiating in the optical is launched during this outburst, the thermal emission of the hot disk remains outshined by the donor.

V4641 Sgr was active also in 1964-1967, that is long time before its main outburst. In this epoch, the activity usually displays low-amplitude fluctuations $(<1 \mathrm{mag})$ on the typical timescale of several weeks. They are independent on the orbital phase and preclude the modulation observed by [2], although their amplitudes are comparable. These fluctuations, including a larger brightening near JD 2438 950, suggest persistent strong activity of the system. The prevailing skewness of the statistical distribution in Figure $1 \mathrm{~g}$ toward the higher brightness indicates faint outbursts.

\section{Acknowledgments}

The support by the grant 205/08/1207 of the Grant Agency of the Czech Republic and the projects D-25-CZ4/08-09 DAAD, PECS 98023 Integral, and PECS 98058 Gaia is acknowledged. This research has made use of observations from the AAVSO International database (Massachusetts, USA). We thank the variable star observers worldwide whose observations made this analysis possible. 


\section{References}

[1]V. P. Goranskij, Variable stars in Sagittarius, Astr. Tsirk 1024 (1978).

[2]V. P. Goranskij, The Orbital V-band Light Curve of V4641 Sagittarii, IBVS 5068 (2001).

[3]D. C. Hannikainen, R. W. Hunstead, D. Campbell-Wilson, K. Wu, D. J. McKay, D. P. Smits, R. J. Sault, Radio Emission from GRO J1655-40 during the 1994 Jet Ejection Episodes, ApJ 540, 521 (2000).

[4]A. Henden, 2008, AAVSO International database, private communication

[5]) R. M. Hjellming, M. P. Rupen, R. W. Hunstead, D. Campbell-Wilson, A. J. Mioduszewski, B. M. Gaensler, D. A. Smith, R. J. Sault, R. P. Fender, R. E. Spencer, et al., Light Curves and Radio Structure of the 1999 September Transient Event in V4641 Sagittarii (=XTE J1819-254=SAX J1819.3-2525), ApJ 544,977 (2000).

[6]C. Lindstrom, J. Griffin, L. L. Kiss, M. Uemura, A. Derekas, Sz. Mészáros, P. Székely, New clues on outburst mechanisms and improved spectroscopic elements of the black hole binary V4641 Sagittarii, MNRAS 363, 882 (2005).

[7]C. B. Markwardt, J. H. Swank, F. E. Marshall, XTE J1819-254, XTE J1743-363, XTE J1710-281, XTE J1723-376, IAUC 7120 (1999).

[8]J. A. Orosz, E. Kuulkers, M. van der Klis, J. E. McClintock, M. R. Garcia, P. J. Callanan, Ch. D. Bailyn, R. K. Jain, R. A. Remillard, A Black Hole in the Superluminal Source SAX J1819.3-2525 (V4641 Sgr), ApJ 555, 489 (2001).

[9]M. Revnivtsev, M. Gilfanov, E. Churazov, R. Sunyaev, Super-Eddington outburst of V4641 Sgr, A\&A 391, 1013 (2002).

[10]T. Shahbaz, E. Kuulkers, On the outburst amplitude of the soft X-ray transients, MNRAS 295, L1 (1998).

[11]V. Šimon, Outburst parameters and the long-term activity of the dwarf nova CH Ursae Majoris, A\&A 354, 103 (2000).

[12]V. Šimon, The properties of outbursts and long-term activity of the soft X-ray transient $4 U$ 1608-52 (QX Nor), A\&A 418, 617 (2004).

[13]R. Stubbings, GM Sagittarii and SAX J1819.3-2525 = XTE J1819-254, IAUC 7253 (1999).

[14]M. Uemura, T. Kato, R. Ishioka, K. Tanabe, S. Kiyota, B. Monard, R. Stubbings, P. Nelson, T. Richards, Ch. Bailyn, R. Santallo, Rapid Optical Fluctuations in the Black Hole Binary V4641 Sagittarii, PASJ 54, L79 (2002).

[15]N. Vogt, The SU UMa stars - an important sub-group of dwarf novae, A\&A 88, 66 (1980). 